Revista de

Contabilidade e

Organizações

www.rco.usp.br
DOI: http://dx.doi.org/10.11606/rco.v10i27.107284
Journal of

Accounting and

Organizations

www.rco.usp.br

\title{
Impactos de outros resultados abrangentes sobre o gerenciamento de resultados
}

\author{
Franciele Wrubela; Vania Regina Morás ${ }^{\mathrm{a}}$; Josiane Brighentia ${ }^{\mathrm{a}}$ Maurício Leite ${ }^{\mathrm{a}}$; Roberto Carlos Klann ${ }^{\mathrm{a}}$ \\ ${ }^{a}$ Universidade Regional de Blumena
}

\section{Informações do Artigo}

Histórico do Artigo

Recebido: 09 de novembro de 2015

Aceito: 17 de agosto de 2016

\section{Palavras-chave:}

Outros Resultados Abrangentes.

Gerenciamento de resultados.

Accruals Discricionários.

\begin{abstract}
Resumo
O estudo objetivou verificar o impacto de Outros Resultados Abrangentes (ORA) na prática de gerenciamento de resultados (GR) nas companhias abertas brasileiras. A pesquisa se caracteriza como descritiva, documental e quantitativa. $O$ período de análise compreende 2010 a 2013. Além do ORA, utilizaram-se como variáveis do GR o Tamanho (LnTam), Endividamento (Endiv), Alavancagem Financeira (AlavFin) e Fluxo de Caixa Operacional (FCO). Como resultado, constatou-se correlação significativa e positiva do ORA com o tamanho das empresas em 2010. Em 2012, ORA apresentou correlação significativa negativa com o FCO e com o Endividamento. O $\mathrm{AD}$ apresentou correlação significativa e negativa com o Endividamento em 2011 e 2012 e com a Alavancagem em 2012, já em 2010 e 2012 apresentou correlação significativa e positiva com a Alavancagem. Conclui-se que não foi possível confirmar que o ORA diminui os níveis de GR e nem que sua divulgação aumenta a transparência das informações contábeis para a redução da assimetria informacional.
\end{abstract}

Copyright (C) 2016 FEA-RP/USP. Todos os direitos reservados

\section{INTRODUÇÃO}

A disponibilização de informação contábil busca auxiliar gestores na condução das organizações e serve de base para tomada de decisão. A divulgação das informações visa ainda a redução da assimetria informacional e, o seu detalhamento atende de maneira favorável aos diversos stakeholders.

A divulgação contábil no Brasil, a partir da adoção de Normas Internacionais de Contabilidade (International Accounting Standards - IFRS), objetivou a redução da assimetria informacional para os usuários, por meio do aumento da transparência. Entretanto, a informação é uma equação composta pelos usuários de um lado e a companhia do outro. Essa equação informacional tem gerado diversas pesquisas, sendo que o presente estudo foi desenvolvido com foco na possibilidade de gerenciamento de resultados por parte das companhias.

Em convergência com as IFRS, desde 2009, no Brasil, adota-se a Divulgação de Resultados Abrangentes com base no Pronunciamento Técnico 26 - CPC 26 (R1). O resultado abrangente corresponde às modificações no patrimônio líquido que não sejam oriundas das transações de capital entre a companhia e seus sócios (CPC 26 (R1)). O conceito de resultado abrangente no país é novo e ainda pouco discutido nas organizações. Este assunto foi objeto de estudo de Lin e Rong (2012) nas empresas chinesas. Os autores testaram a hipótese de que quanto maior a qualidade da divulgação das informações, menor é a assimetria de informação. Dessa forma, seriam menores as chances de gerenciamento de resultados. Por outro lado, conforme Lin e Rong (2012), quanto menor a qualidade da divulgação das informações, maior a diferença do grau de assimetria de informação entre os investidores e as companhias com ações negociadas em bolsa de valores e, consequentemente, maiores as chances para o gerenciamento de resultados.

A Demonstração de Resultados Abrangentes foi incorporada ao conjunto completo obrigatório das demonstrações contábeis em setembro de 2009, por meio da aprovação do Pronunciamento Técnico CPC 26, pela

\footnotetext{
Autor Correspondente: Tel (47) 3321-0200

E-mail: franciele_wrubel@yahoo.com.br (F. Wrubel); vaniar.moras@gmail.com (V. R. Morás); josi_brig@unochapeco.edu.br (J. Brighenti);

mauricio.leite@ymail.com (M. Leite); rklann@furb.br (R. C. Klann)

Universidade Regional de Blumenau - R. Antônio da Veiga, 140 - Itoupava Seca, Blumenau - SC, 89012-900, Brasil.
} 
Comissão de Valores Mobiliários (CVM), o qual foi emitido pelo Comitê de Pronunciamentos Contábeis (CPC). Assim, o foco que se propôs abordar nessa pesquisa foi o lucro abrangente e o gerenciamento de resultados no Brasil.

O gerenciamento dos resultados contábeis (earnings management) incorre da alteração intencional de resultados contábeis, visando atender motivações particulares (MARTINEZ, 2006). A alteração de resultados, por meio dos Accruals Discricionários (AD), que são contas de resultado que geraram o lucro, mas que não implicam em necessária movimentação de disponibilidades, pode ser considerado gerenciamento de resultados. Os indicadores de desempenho podem influenciar na decisão de aumentar os AD e gerenciar resultados, ou reduzir os accruals negativos, implicando na redução do lucro líquido (IUDÍCIBUS; LOPES, 2004).

Partindo do pressuposto de que em algumas situações o gestor pode fazer escolhas contábeis no sentido de reconhecer o efeito de determinadas transações no resultado ou no Patrimônio Líquido (PL), como no caso de alguns instrumentos financeiros, é possível supor que este grupo do PL (Outros Resultados Abrangentes - ORA) possa ser utilizado para gerenciar o lucro das empresas, tanto para aumentá-lo, como para diminuí-lo. Desta forma, o problema de pesquisa que se coloca é: qual é o impacto de Outros Resultados Abrangentes no gerenciamento de resultados nas companhias brasileiras? Assim, o objetivo do estudo é analisar o impacto de Outros Resultados Abrangentes no gerenciamento de resultados nas companhias brasileiras. A pesquisa justifica-se pelo fato de analisar o detalhamento dos resultados abrangentes das companhias; o quanto os valores de ORA representam, considerando que a adoção da prática contábil de registro de ORA altera o valor do lucro; e qual é a influência desses valores no accrual discricionário, proxy de gerenciamento de resultados.

A influência do lucro abrangente na distribuição de dividendos foi foco de Kochiyama e Itou (2013). Coelho e Carvalho (2007) fizeram uma análise conceitual de lucro abrangente e lucro operacional corrente. Mazzioni, Oro e Scarpin (2013) utilizaram o lucro líquido e o resultado abrangente para avaliá-los como medidas preditivas do desempenho das companhias do segmento elétrico brasileiro. Entretanto, são encontrados poucos estudos para analisar os efeitos e influências especificamente dos ORA.

As pesquisas de Biddle e Choi (2006), Coelho e Carvalho (2007), Kanagaretnam, Mathieu e Shehata (2009), Macedo, Vilamaior e Pinheiro (2010) e Cahan et al. (2000) não encontraram evidências para sustentar que o resultado abrangente tem maior capacidade explicativa do desempenho empresarial em relação ao lucro. Assim, constata-se que os estudos ainda não são conclusivos quanto à utilização e divulgação de lucro abrangente ou ORA na redução da assimetria da informação e no gerenciamento de resultados. Dessa forma, o artigo proposto visa preencher essa lacuna de pesquisa por meio da análise desse impacto de ORA nos AD de companhias listadas na BMF\&Bovespa, no período de 2010, ano de adoção da divulgação da DRA, até 2012, tornando possível contribuir com as discussões citadas sobre o assunto.

\section{REFERENCIAL TEÓRICO}

Nesta revisão de literatura inicialmente são apresentados conceitos de Resultados Abrangentes, seguido da discussão sobre Disclosure e Assimetria da Informação, e ainda aspectos relacionados ao Gerenciamento de Resultados.

\subsection{Resultado abrangente}

A informação contábil busca auxiliar os gestores, servindo de base para a tomada de decisão. Pinheiro, De Macedo e Vilamaior (2012) destacam que na busca pela evolução e aprimoramento da informação contábil, o Financial Accounting Standard Board - FASB (1997) emitiu o Pronunciamento 130, o qual determina que alguns ganhos, perdas, receitas e despesas não realizados devem ser registrados no PL das empresas em rubrica específica.

O Brasil, em convergência com as Normas Internacionais de Contabilidade, adota a elaboração da Divulgação de Resultados Abrangentes desde 2009, com base no Pronunciamento Técnico CPC 26 - CPC 26 (R1). Esse Pronunciamento, no item 7, trata como resultado abrangente a "mutação que ocorre no patrimônio líquido durante um período que resulta de transações e outros eventos que não sejam derivados de transações com os sócios na sua qualidade de proprietários". 
Traz ainda que o resultado abrangente compreende todos os componentes da demonstração do resultado e da demonstração dos ORA. Por sua vez, os ORA compreendem itens de receita e de despesa, incluindo os ajustes de reclassificação, não reconhecidos na demonstração de resultado. Os ORA incluem variações na reserva de reavaliação; ganhos e perdas; e parcela efetiva de ganhos ou perdas advindas de instrumentos de hedge. Vale salientar que para cada um desses componentes há um pronunciamento específico que determina as regras.

Na concepção de Hendriksen e Van Breda (1999, p. 208), o lucro abrangente corresponde à “variação total do valor do capital, reconhecido pelo registro de transações ou pela reavaliação da empresa durante um período determinado, excetuando-se os pagamentos de dividendos e as transações de aumento ou redução de capital". Dessa forma, o lucro abrangente é mais amplo do que o lucro líquido, pois inclui outras variações dos ativos líquidos reconhecidos no período, que podem originar-se de valores de mercado de títulos negociáveis, além de ajustes de valores oriundos da convergência em moeda estrangeira. (HENDRIKSEN; VAN BREDA, 1999).

Os pronunciamentos, interpretações e orientações reguladoras da atividade contábil no Brasil determinam que a elaboração da Demonstração do Resultado Abrangente (DRA) seja realizada com base na soma do resultado líquido apresentado na Demonstração do Resultado do Período (DRE), com os ORA (IUDÍCIBUS et al., 2010). Desta forma, o total do resultado abrangente corresponde às modificações no PL que não sejam oriundas de transações entre a empresa e seus sócios.

Quanto à estrutura, o item 82A do CPC 26 (R1) determina que a DRA deve no mínimo incluir o resultado líquido resultante do período, cada item dos ORA que devem ser classificados conforme sua natureza, a parcela dos ORA oriundos de empresas investidas reconhecida pela equivalência patrimonial e o resultado abrangente do período. Sugere ainda que a DRA seja apresentada dentro da Demonstração das Mutações do Patrimônio Líquido (DMPL), ou ainda, por meio de relatório próprio. Quando a divulgação ocorrer em demonstrativo próprio, Iudícibus et al. (2010) salienta que deverá ter como valor inicial o resultado líquido do período apurado na DRE, seguido dos ORA.

Assim, os ORA pertencem à mutação do Patrimônio líquido (PL) das companhias, inclusive a conta Ajuste de Avaliação Patrimonial do PL pode representar esses valores. Nessa conta, registram-se contrapartidas de aumentos ou diminuições de valor atribuído a elementos do ativo e do passivo, em decorrência de sua avaliação a valor justo, enquanto não computadas no resultado do exercício sendo que, os valores registrados nessa conta deverão ser transferidos para o resultado do exercício à medida que os ativos e passivos forem sendo realizados (IUDÍCIBUS et al., 2010).

Conforme o ICPC 10, essa conta foi contrapartida de eventuais ajustes nas contas de ativo imobilizado na situação em que a entidade pôde adotar a opção de atribuir um valor justo inicial (utilização do conceito de custo atribuído - deemed cost) ao ativo imobilizado nos termos dos itens 21 a 29 da referida Interpretação, em função da mudança da prática contábil brasileira e adoção inicial em 2010, do processo de convergência referente aos CPC 27 (Imobilizado) e CPC 28 (Propriedade para Investimento). Dessa forma, na concepção de Epstein, Nach e Bragg (2009), o Lucro Abrangente demonstra melhor o desempenho das empresas que o Lucro Líquido, pois inclui todas as alterações patrimoniais do período, exceto aquelas oriundas de investimentos dos proprietários e as distribuições para estes.

Uma importante diferença entre lucro operacional e o lucro abrangente, segundo Hendriksen e Van Breda (1999), está na divulgação do resultado líquido. O lucro operacional enfatiza a eficiência operacional corrente da empresa e pode ser utilizado para a predição do desempenho e da capacidade de geração de resultados futuros. Já os defensores do conceito abrangente do lucro ressaltam que, tanto a eficiência operacional da empresa quanto a predição de desempenho futuro podem ser melhor elaboradas se tomarem por base a experiência histórica completa da empresa em uma série de anos, em vista de que o lucro líquido operacional é baseado em um único período e pode estar sujeito à verificação em datas futuras.

\subsection{Gerenciamento de resultados}

O gerenciamento de resultados, conforme Healy e Wahlen (1999), acontece quando utiliza-se de julgamentos sob a informação financeira e sobre as atividades operacionais, para alterar informações financeiras ou iludir intencionalmente investidores sobre o desempenho econômico da companhia, ou para influenciar resultados contratuais que dependam de números contábeis. Para Schipper (1989), o gerenciamento de resultados é a intervenção, realizada propositalmente, no processo de evidenciação externa, com a intenção de obter algum ganho privado. 
Martinez (2006) destaca que a gestão direciona os resultados com propósitos específicos e não como expressão da realidade do negócio. No entanto, o gerenciamento dos resultados contábeis não se constitui fraude contábil, pois se opera dentro dos limites que prescreve a legislação contábil. Um dos produtos mais importantes da contabilidade é o resultado, pois por meio deste pode-se avaliar o desempenho das empresas. Porém, esse resultado pode sofrer ajustes contábeis de natureza discricionária, sem haver qualquer relação com o negócio empresarial. Tais ajustes levam os executivos a gerenciar os resultados contábeis de forma desejada.

Na tomada de decisões sobre o reconhecimento das despesas e a variabilidade dos resultados contábeis, Martinez (2006) considera que as despesas de depreciação, despesas com provisão para devedores duvidosos e as receitas não-operacionais reduzem a variabilidade dos resultados contábeis. Já as variações na amortização de ágio e deságio de investimentos, provisões para perdas em investimentos e reservas de reavaliação relacionam-se com ações para reduzir a variabilidade dos resultados.

Todas as contas de resultado que entraram no cômputo do lucro, mas que não implicam em necessária movimentação de disponibilidades, são conhecidas como accruals, que em sentido amplo, consiste na diferença entre o lucro líquido e o fluxo de caixa operacional líquido. Tais accruals podem ser utilizados para fins de gerenciamento de resultados. Os accruals podem ser discricionários (discretionary accruals) e não discricionários (non discretionary accruals). Os últimos refletem a realidade do negócio, enquanto os primeiros são artificiais, com o único propósito de gerenciar o resultado contábil (earnings management) (MARTINEZ, 2008).

Os AD são proxys do gerenciamento de resultados contábeis, o desafio é estimar o seu valor, que pode ser positivo ou negativo. Representam a forma como a empresa gerencia seus resultados, para melhorá-los ou piorá-los. É possível estimar o valor dos accruals totais a partir das variações dos itens específicos no balanço patrimonial, as contas de resultados nem sempre discriminam exatamente o seu montante (MARTINEZ, 2008).

Existem diversas modalidades de gerenciamento de resultados, dependendo das motivações que estão envolvidas no processo. Martinez (2006) destaca as três principais, conforme apresentadas na Figura 1.

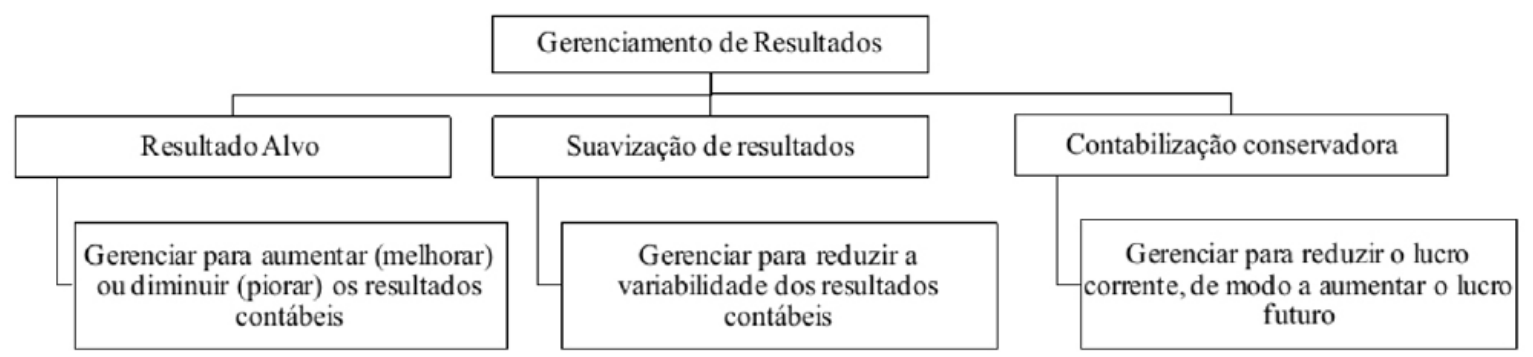

Figura 1. Modalidades de gerenciamento de resultados Fonte: Adaptado de Martinez (2006).

Conforme se observa na Figura 1, a modalidade resultado alvo consiste em gerenciar o resultado para atingir determinadas metas de lucro, independente do resultado do período. Na modalidade suavização de resultados o objetivo é reduzir a variabilidade dos resultados e estabilizá-los em determinado patamar. Por fim, a contabilização conservadora consiste na empresa piorar seu resultado atual para melhorar o resultado futuro. Como exemplo, pode-se citar a antecipação e reconhecimento de despesas no período, as quais poderiam ser reconhecidas em períodos futuros.

As empresas podem adotar diferentes modelos para detectar práticas de gerenciamento de resultados, como: Healy (1985), DeAngelo (1986), Dechow e Sloan ou modelo Setorial (1991), Jones (1991), Dechow, Sloan e Sweeney (1995) (ou Jones Modificado), Kang e Sivaramakrishnan (1995) ou modelo KS, Pae (2005), Leuz, Nanda e Wysocki (2003), Barth, Landsman e Lang (2008).

Um dos modelos mais utilizados na literatura, principalmente em estudos com empresas brasileiras, é o modelo KS. Esse modelo inclui as despesas operacionais, bem como trata das contas a receber para lidar com eventuais problemas associados à manipulação nas receitas (MARTINEZ, 2008). Para corrigir o problema de simultaneidade, é empregada a metodologia de variáveis instrumentais. O modelo KS opera diretamente com as contas do balanço patrimonial, evitando-se o indesejável problema de comparar valores em moeda de diferentes períodos. Esse detalhe metodológico ganha relevância no Brasil, quando se considera o aspecto inflacionário, que comprometeria a comparabilidade entre dois exercícios. Conforme Martinez (2008) e Thomas e Zhang (2001), não existe modelo perfeito, mas o modelo KS é o que proporciona os melhores resultados. Diante disto, determinou-se os AD com a utilização do modelo KS. 


\subsection{Estudos relacionados}

Com base na apresentação e contextualização realizada sobre os temas resultado abrangente e gerenciamento de resultados, construiu-se o Quadro 1, que apresenta a descrição de alguns principais estudos realizados sobre os assuntos.

\begin{tabular}{cl}
\hline Autores & \multicolumn{1}{c}{ Principais resultados } \\
\hline & $\begin{array}{l}\text { Não encontrou evidência clara de que o resultado global (resultado } \\
\text { abrangente) está mais fortemente associado, em termos de poder explicativo, }\end{array}$
\end{tabular}

ao retorno do que o lucro líquido.

Os achados indicam que a abordagem de resultado abrangente fornece mais utilidade de decisão para o conteúdo de informação, capacidade de previsão e contrato de remuneração de executivos. Definições diferentes de

Biddle e Choi (2006) renda fornecem diferentes utilidades de decisão em diferentes aplicações, e, que é uma decisão útil divulgar separadamente componentes do resultado abrangente, corroborando o posicionamento do IASB na exigência da divulgação da informação.

Realizaram análise conceitual de lucro abrangente e lucro operacional corrente buscando evidências em empresas do setor financeiro brasileiro, referente aos períodos entre 2001 e 2004. Os achados indicam diferenças expressivas nos lucros divulgados pelos dois conceitos nas demonstrações contábeis, não havendo evidenciação das diferenças encontradas entre o lucro reportado e o incremento do PL, nem mesmo em notas explicativas.

Estudo em 391 companhias chinesas em 2009, constataram que a divulgação de ORA é negativamente relacionada com o gerenciamento de resultados nestas empresas. Dessa forma, acredita-se que a divulgação de outros resultados abrangentes pode reduzir o gerenciamento de resultados, a fim de fazer o público entender melhor o desempenho de uma determinada empresa e reduzir a assimetria de informação.

Os resultados demonstram que o eventual reconhecimento dos ORA no

Pinheiro, De Macedo e Vilamaior (2012) resultado causaria maior volatilidade no lucro líquido das empresas, sem tendência definida, no sentido de aumentá-lo ou diminuí-lo.

Não há evidência de que o resultado abrangente de t0 possui capacidade

Mazzioni, Oro e Scarpin (2013) preditiva superior ao lucro líquido de t0 para prever os resultados de t1 para as empresas do setor elétrico de 2010 e 2011.

O coeficiente de variação para resultados abrangentes é significativamente Kochiyama e Itou (2013) mais elevado do que os outros rendimentos contábeis. ORA negativos têm um impacto nos dividendos, ou seja, conduz a dividendos inferiores.

Quadro 1. Estudos anteriores

Fonte: Dados da pesquisa.

No Quadro 1 encontram-se sintetizados os estudos encontrados entre 1994 a 2013 relacionados com o tema pesquisado. Evidências de que o resultado abrangente tem maior capacidade explicativa do que o lucro, podem ser encontradas nos estudos de Dhaliwal, Subramanyam e Trezevant (1999), Newberry (2003), Pinto (2005), Soutes e Schvirck (2006), Gallon et al. (2009), Resende, Pinheiro e Maia (2011). De outro modo, as investigações de Biddle e Choi (2006), Coelho e Carvalho (2007), Kanagaretnam, Mathieu e Shehata (2009), Macedo, Vilamaior e Pinheiro (2010) e Cahan et al. (2000) não encontraram evidências para sustentar que o resultado abrangente tem maior capacidade explicativa do desempenho empresarial em relação ao lucro.

Dessa forma foi possível constatar a existência da lacuna de pesquisa referente ao impacto dos ORA no gerenciamento de resultados. Isso ocorre pelo fato de que os estudos não são conclusivos quanto à influência positiva ou negativa dos diferentes conceitos de lucro sobre os indicadores de desempenho, na melhor explicação do lucro, na capacidade preditiva destes, na redução da assimetria da informação com a qualidade da informação divulgada, bem como no nível de gerenciamento de resultados. 


\section{ASPECTOS METODOLÓGICOS DA PESQUISA}

Neste capítulo são apresentadas a classificação metodológica da pesquisa, a população e amostra do estudo, bem como os procedimentos para coleta e análise dos dados.

\subsection{Método de Pesquisa}

Quanto à sua natureza, a pesquisa pode ser classificada como aplicada, pois objetiva identificar um determinado comportamento (gerenciamento de resultados) praticado pelas companhias listadas na BM\&FBovespa. No que se refere aos seus objetivos, a pesquisa classifica-se como descritiva, por relacionar o gerenciamento de resultados com os resultados abrangentes. Quanto à abordagem do problema, a pesquisa revestese de características quantitativas, já que se utilizou de técnicas estatísticas na coleta e no tratamento dos dados. Quanto aos procedimentos técnicos, a pesquisa é documental.

\subsection{População e amostra}

A população que compõem este estudo é compreendida pelas companhias abertas listadas na BM\&FBovespa durante os anos de 2010 a 2013. Para formar a amostra, as companhias deveriam estar listadas no período de 2010 e possuir valores em Resultados Abrangentes e demais informações necessárias para a coleta de dados. A amostra inicial foi composta por 77 companhias. Desta amostra foram excluídas as instituições financeiras e de seguros, pois possuem regulamentações econômicas e contábeis significativamente diferentes dos demais ramos de atividades econômicas, além das companhias que não possuíam informações suficientes para gerar o $\mathrm{AD}$, variável dependente das regressões realizadas. Considerou-se também a exclusão as empresas outliers, ou seja, as empresas com resíduo maior que quatro vezes o valor do desvio padrão do resíduo de todas as empresas. As companhias outliers foram retiradas para resolver problemas de heteroscedasticidade, conforme recomenda Fávero et al., 2009. Uma composição da amostra final está descrita na Tabela 1.

Tabela 1. População e amostra

\begin{tabular}{lcccc}
\hline \multicolumn{1}{c}{ Itens } & $\mathbf{2 0 1 0}$ & $\mathbf{2 0 1 1}$ & $\mathbf{2 0 1 2}$ & $\mathbf{2 0 1 3}$ \\
\hline População total & 77 & 77 & 77 & 77 \\
(-) companhias sem valores de Resultados abrangentes & & 25 & 24 & 60 \\
(-) companhias sem valores em Accrual Discricionário & - & 4 & 8 & 4 \\
(-) companhias sem valores em outras variáveis & - & - & 1 & - \\
(=) Amostra parcial & 77 & 48 & 44 & 13 \\
(-) instituições financeiras e de seguros & 2 & 2 & 2 & 13 \\
(-) outliers & 1 & - & - & - \\
(=) Amostra final & $\mathbf{7 4}$ & $\mathbf{4 6}$ & $\mathbf{4 2}$ & $\mathbf{0}$ \\
\hline
\end{tabular}

Fonte: Dados da pesquisa.

A quantidade de observações (N) para cada ano foi de: 74 companhias em 2010; 46 companhias em 2011 e 42 em 2012. Já no ano de 2013 não foi possível obter amostra em razão das exclusões realizadas. Conforme apresentado na Tabela 1, comparando com os anos anteriores, em 2013 a quantidade de Resultado Abrangente (variação da conta Ajuste de Avaliação Patrimonial) divulgado diminuiu, sendo que as empresas que mais divulgaram em 2013 foram as instituições financeiras. Apresentam-se na sequência os detalhes sobre a coleta e análise dos dados dessas empresas pertencentes à amostra.

\subsection{Coleta e análise dos dados}

A coleta dos dados foi realizada no banco de dados Economática ${ }^{\circledR}$. Tomou-se como base o período de quatro 
anos (2010 a 2013), considerou-se a disponibilidade dos dados com o intuito de não reduzir de maneira substancial a amostra, dado o número de variáveis independentes coletadas. Optou-se por não incluir dados anteriores ao ano de 2010, devido ao fato de que a Demonstração dos Resultados Abrangentes não era exigida pelas normas do Conselho Federal de Contabilidade antes desse período, já o ano de 2013 não foi possível operacionalizar em razão da ausência de dados das empresas que compõe a amostra.

Utilizou-se o Modelo KS de Kang \& Silvaramakrishnan (1995), pois tal modelo é o mais apropriado para o contexto brasileiro. Segundo Martinez (2008) e Thomas e Zhang (2001), o Modelo KS é um dos melhores modelos de avaliação de gerenciamento de resultados, pois opera diretamente nas contas do Balanço Patrimonial, na tentativa de explicar a dimensão dos Accruals Totais. Os AD são computados como resíduos da equação 2 , nos seguintes termos:

$$
\begin{gathered}
A T_{i t}=\emptyset_{0}+\emptyset_{1}\left[\delta_{1} \operatorname{Rec}_{i t}\right]+\emptyset_{2}\left[\delta_{2} \operatorname{Desp}_{i t}\right]+\emptyset_{3}\left[\delta_{3} \text { At.Imob } \text { Im }_{i t}\right]+\varepsilon_{i t} \\
A D_{i t}=A T_{i t}-\left\{\varnothing_{0}+\emptyset_{1}\left[\delta_{1} \operatorname{Rec}_{i t}\right]+\varnothing_{2}\left[\delta_{2} \text { Des }_{i t}\right]+\emptyset_{3}\left[\delta_{3} A t . I m o b_{i t}\right]\right\}
\end{gathered}
$$

As variáveis $\operatorname{Rec}_{\mathrm{it}}, \operatorname{Desp}_{\mathrm{it}}, \mathrm{At}$ Imob ${ }_{\mathrm{it}}$ são escaladas em termos de ativos totais.

$\mathrm{AT}_{\mathrm{it}}=$ Acumulações Totais $=(\mathrm{CGL}-$ Depreciação \& Amortização $)$

$\operatorname{Rec}_{i t}=$ Receita Líquida (excluindo tributação)

Desp $_{\mathrm{it}}=$ Custos de Despesas Operacionais antes da Depreciação \& Amortização

CGL = Capital de Giro Líquido excluindo as disponibilidades, financiamentos de curto prazo e provisão de Impostos a Pagar At.Imob : $_{\text {: }}$ Ativo Imobilizado e Ativo Diferido.

$$
\begin{aligned}
& \text { C.Receber }{ }_{\mathrm{i}, \mathrm{t}-1}=\text { Contas a Receber no período } t-1 \\
& \text { DEPREC }_{\mathrm{i}, \mathrm{t}-1}=\text { Despesas de Depreciação } \\
& =\mathrm{C} \cdot \operatorname{Receb}_{\mathrm{i}, \mathrm{t}-1} / \operatorname{Rec}_{\mathrm{i}, \mathrm{t}-\mathrm{I}} \text {, onde AR (contas a receber) } \\
& =\left(\Delta \mathrm{CG}-\mathrm{CRec}_{\mathrm{it}}\right) / \mathrm{Desp}_{\mathrm{i}, \mathrm{t}-1} \\
& =\mathrm{DEPREC}_{\mathrm{i}, \mathrm{t}-1} / \mathrm{A} \cdot \operatorname{Imob}_{\mathrm{i}, \mathrm{t}-1}
\end{aligned}
$$

Martinez (2008) conceitua que o accrual total constitui a variação do capital de giro líquido excluído das disponibilidades e financiamentos de curto prazo, que é subtraído do total da depreciação e amortização, sendo esse saldo final medido em termos de porcentagem dos ativos totais. Caso existam provisões tributárias, também devem ser excluídas do cômputo do accrual total.

Conceitualmente, um AD positivo significa que a companhia está gerenciando seus resultados para aumentá-los. Por outro lado, um AD negativo implica que a organização adotam práticas contábeis que objetivam reduzir o lucro líquido. Com esta base conceitual, buscou-se relacionar os accruals com as informações das variáveis relacionadas no Quadro 3, por meio da regressão de dados em painel, conforme a equação 3:

$$
A D=\beta_{0}+\beta_{1} L n_{-} T a m+\beta_{2} \text { Endiv }+\beta_{3} \text { AlavFin }+\beta_{4} F C O+\beta_{5} O R A+\varepsilon_{i t}
$$

Como variável independente ou explicativa utilizou-se os ORA. As variáveis relacionadas ao Logaritmo Natural Tamanho da companhia (LnTam), Endividamento (Endiv), Alavancagem Financeira (AlavFin) e Fluxo de Caixa Operacional (FCO) são utilizadas como variáveis de controle, assim como aponta o estudo realizado por Lin e Rong (2012). Conforme esses autores, o efeito esperado dessas variáveis no grau de gerenciamento de resultados é negativo para o tamanho da empresa, ORA e para o FCO; e positivo para AlavFin e Endiv.

Os autores explicam que, para evitar custos políticos, gestores muitas vezes tentam reduzir os lucros divulgados para apresentar uma imagem de organizações sem fins lucrativos perante o público. Tamanho da companhia é amplamente adotado para substituir custo político em estudos relacionados ao custo político. Por isso quanto maior a empresa, menor é o grau de gerenciamento de resultados.

Em relação ao fluxo, o AD é adotado como proxy de gerenciamento de resultados. Considerando um determinado lucro líquido ou o lucro operacional, quanto maior o fluxo de caixa, menor é o accrual total, portanto, menores os AD, por isso espera-se um coeficiente negativo para o FCO. Para Defond e Park (1997), a alavancagem financeira refere-se de certa forma a accruals. Especificamente, quanto maior o nível da alavancagem, menores 
são os níveis de ativos e passivos e maiores os accruals.

O Quadro 2 apresenta a descrição da variável dependente e as variáveis independentes, bem como a literatura que suporta sua utilização.

\begin{tabular}{|c|c|c|c|c|}
\hline Variável & Sigla & Nome da Variável & Explicação & Autores \\
\hline 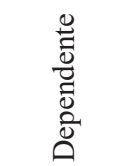 & $\mathrm{AD}$ & $\begin{array}{c}\text { Accruals } \\
\text { Discricionários }\end{array}$ & $\begin{array}{l}\text { Proxy de gerenciamento } \\
\text { de resultados }\end{array}$ & $\begin{array}{l}\text { Paulo e Leme (2009); Lin e } \\
\text { Rong (2012) }\end{array}$ \\
\hline \multirow{5}{*}{ 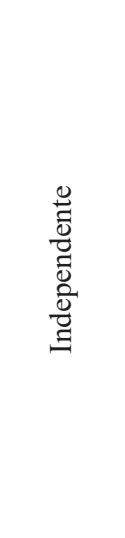 } & LnTam & Tamanho & Logaritmo do ativo & Lin e Rong (2012). \\
\hline & Endiv & Endividamento & $\frac{\mathrm{PC}+\mathrm{PNC}}{\text { Ativo Total }}$ & $\begin{array}{c}\text { Martinez (2001); Coelho e } \\
\text { Lopes (2007). }\end{array}$ \\
\hline & AlavFin & $\begin{array}{l}\text { Alavancagem } \\
\text { Financeira }\end{array}$ & $\frac{\mathrm{PC}+\mathrm{PNC}}{\text { Patrimônio Líquido }}$ & $\begin{array}{l}\text { Defond e Park (1997); Coelho } \\
\text { e Lopes (2007); Klann (2011); } \\
\text { Lin e Rong (2012). }\end{array}$ \\
\hline & $\mathrm{FCO}$ & $\begin{array}{l}\text { Fluxo Caixa } \\
\text { Operacional }\end{array}$ & $\frac{\text { FCO }}{\text { Ativo Total }}$ & $\begin{array}{l}\text { Paulo e Leme (2009); Klann } \\
\text { (2011); Lin e Rong (2012). }\end{array}$ \\
\hline & ORA & $\begin{array}{l}\text { Outros Resultados } \\
\text { Abrangentes }\end{array}$ & $\begin{array}{l}\text { Ajuste de avaliação } \\
\text { Patrimonial (PL) } \\
\frac{\left(\text { Ano }_{t}-\text { Ano }_{\mathrm{t}-1}\right)}{\text { Ativo Total }}\end{array}$ & $\begin{array}{c}\text { Lin e Rong (2012); Pinheiro, } \\
\text { De Macedo e Vilamaior } \\
\text { (2012). }\end{array}$ \\
\hline
\end{tabular}

Quadro 2. Variáveis do estudo

Fonte: Dados da pesquisa.

Lin e Rong (2012) utilizaram duas variáveis alternativas (proporções de ativos e dívidas) para a variável referente à alavancagem financeira. Já neste estudo utilizou-se apenas a alavancagem financeira (AlavFin). Quanto à variável ORA, utilizou-se a diferença entre os saldos anuais da conta de Ajustes de Avaliação Patrimonial (Ano $\mathrm{t}$ - Ano $\mathrm{t}-1$ ), por representar as mutações que afetam o PL, mas que não são ou não foram ainda reconhecidas no resultado do exercício.

Para analisar os dados, inicialmente verificou-se a normalidade da amostra pelo teste KolmogovovSimirnov (KS), no qual constatou-se que os dados são normais. Foram realizados testes de heteroscedasticidade com os dados das variáveis do Quadro 2, e os resultados apresentaram-se adequados. Para fazer a análise de dados em painel se fez o teste de autorrelação dos resíduos (Durbin-Watson) e de multicolinearidade (VIF). Os resultados foram adequados e estão apresentados e discutidos junto às demais análises dos dados. Além da análise multivariada de dados em painel, os dados das variáveis também foram analisados por meio da estatística descritiva e da análise de correlação de Pearson, conforme se apresenta na sequência sobre a análise dos resultados da pesquisa.

\section{DESCRIÇÃO E ANÁLISE DOS RESULTADOS}

Esta seção apresenta os resultados obtidos a partir do que estabeleceu-se nos objetivos da pesquisa está dividida em três subseções. A primeira refere-se à análise descritiva, seguida da análise estatística univariada e por fim análise de regressão de dados em painel.

\subsection{Análise descritiva}

A Tabela 2 apresenta as estatísticas descritivas para as variáveis dependente e independentes do modelo: Accrual Discricionário (AD); Tamanho da companhia (LnTam); Endividamento (Endiv), Alavancagem Financeira 
(AlavFin), Fluxo de Caixa Operacional (FCO) e Outros Resultados Abrangentes (ORA).

Tabela 2. Estatística descritiva

\begin{tabular}{|c|c|c|c|c|c|c|}
\hline \multirow{2}{*}{ Ano } & \multirow{2}{*}{ Variáveis } & \multicolumn{5}{|c|}{ Estatística descritiva } \\
\hline & & $\mathrm{N}$ & Mínimo & Máximo & Média & Desvio padrão \\
\hline \multirow{6}{*}{2010} & $\mathrm{AD}$ & \multirow{6}{*}{74} & $-0,865$ & 1,844 & 0,511 & 0,387 \\
\hline & LnTam & & 11,979 & 20,069 & 14,928 & 1,577 \\
\hline & Endiv & & 0,016 & 1,645 & 0,572 & 0,232 \\
\hline & AlavFin & & $-33,600$ & 4,500 & 0,271 & 6,129 \\
\hline & FCO & & $-0,104$ & 0,288 & 0,063 & 0,071 \\
\hline & ORA & & $-0,249$ & 0,111 & 0,001 & 0,036 \\
\hline \multirow{6}{*}{2011} & $\mathrm{AD}$ & \multirow{6}{*}{46} & $-0,166$ & 1,476 & 0,707 & 0,277 \\
\hline & LnTam & & 12,179 & 20,211 & 15,004 & 1,699 \\
\hline & Endiv & & 0,093 & 1,128 & 0,582 & 0,225 \\
\hline & AlavFin & & $-33,319$ & 21,498 & 1,886 & 6,964 \\
\hline & FCO & & $-0,182$ & 0,172 & 0,054 & 0,064 \\
\hline & ORA & & $-0,050$ & 0,442 & 0,010 & 0,066 \\
\hline \multirow{6}{*}{2012} & $\mathrm{AD}$ & \multirow{6}{*}{42} & $-0,016$ & 1,593 & 0,798 & 0,328 \\
\hline & LnTam & & 12,350 & 20,334 & 15,282 & 1,751 \\
\hline & Endiv & & 0,062 & 1,896 & 0,643 & 0,316 \\
\hline & AlavFin & & $-9,449$ & 26,358 & 2,864 & 5,565 \\
\hline & FCO & & $-0,082$ & 0,264 & 0,048 & 0,068 \\
\hline & ORA & & $-0,372$ & 0,004 & $-0,015$ & 0,063 \\
\hline
\end{tabular}

Fonte: Dados da Pesquisa.

Em relação às variáveis apresentadas na Tabela 2, constatou-se que possuem elevados valores de desvio padrão, o que permite constatar instabilidades quanto às variações. Aquelas que apresentam desvio padrão superior à média, em 2010, 2011 e 2012 foram a AlavFin, FCO e ORA. Foi observado aumento da média anual dos valores de $\mathrm{AD}$ nos anos de 2010 a 2012. A média é positiva em todos os anos e indica a ocorrência de gerenciamento para aumento dos lucros. O ano de 2010 teve o menor mínimo $(-0,865)$ entre os anos e também o maior máximo $(1,844)$, sendo, portanto, o ano de menor estabilidade na comparação de AD entre as companhias.

Os ORA das companhias analisadas são os valores com maior instabilidade entre os anos, referentes aos valores mínimos, máximos, média e desvio-padrão. Considerando a média dessa variável, observa-se que em 2011 foram registrados maiores valores positivos de outros resultados abrangentes $(0,010)$. Nos demais anos, os valores médios foram menores e chegaram a ser negativos em 2012 (-0,015). O ano de 2010 foi o período que refletiu a variação dos ORA referente ao custo atribuído (deemed cost), conforme o ICPC 10.

Quanto à variável LnTam, observa-se que houve aumento no tamanho das companhias ao longo do período. Constatou-se ainda redução da média do valor do FCO no período. O valor máximo dessa variável teve uma redução de 2010 para 2011 e aumento de 2011 para 2012. Quanto à variável Endiv, percebe-se que a média aumentou desde 2010 até 2012.

Ao tratar da AlavFin, que representa o nível de recursos financeiros oriundos de capital de terceiros de longo prazo em relação ao capital próprio, percebe-se um aumento consecutivo no período. A discussão dos resultados encontrados para a correlação das variáveis descritas é apresentada a seguir.

\subsection{Análise estatística univariada}

Por meio do programa estatístico SPSS ${ }^{\circledR}$ elaborou-se a Matriz de Correlação de Pearson das variáveis. A Tabela 3 apresenta a correlação entre as variáveis estudadas conforme o constructo da pesquisa. 
Tabela 3. Correlações entre as variáveis

\begin{tabular}{|c|c|c|c|c|c|c|c|c|c|c|c|c|c|c|c|c|c|c|}
\hline Var & \multicolumn{3}{|c|}{$\mathrm{AD}(1)$} & \multicolumn{3}{|c|}{ Ln Tam (2) } & \multicolumn{3}{|c|}{ Endiv (3) } & \multicolumn{3}{|c|}{ AlavFin (4) } & \multicolumn{3}{|c|}{$\mathrm{FCO}(5)$} & \multicolumn{3}{|c|}{ ORA (6) } \\
\hline Ano & 10 & 11 & 12 & 10 & 11 & 12 & 10 & 11 & 12 & 10 & 11 & 12 & 10 & 11 & 12 & 10 & 11 & 12 \\
\hline $\mathrm{N}$ & 74 & 46 & 42 & 74 & 46 & 42 & 74 & 46 & 42 & 74 & 46 & 42 & 74 & 46 & 42 & 74 & 46 & 42 \\
\hline (1) & 1 & 1 & 1 & $-0,11$ & $-0,11$ & $-0,03$ & $-0,19$ & $-0,54^{*}$ & $-0,66^{*}$ & $0,36^{*}$ & $0,42^{*}$ & $-0,31$ & 0,19 & 0,20 & $-0,21$ & $-0,11$ & $-0,13$ & 0,21 \\
\hline (a) & & & & 0,33 & 0,48 & 0,85 & 0,10 & 0,00 & 0,00 & 0,00 & 0,00 & 0,05 & 0,10 & 0,18 & 0,19 & 0,33 & 0,40 & 0,17 \\
\hline (2) & & & & 1 & 1 & 1 & 0,10 & 0,10 & $-0,06$ & 0,16 & 0,15 & 0,10 & 0,01 & 0,12 & $-0,07$ & $0,31 *$ & $-0,13$ & 0,27 \\
\hline (a) & & & & & & & 0,40 & 0,53 & 0,72 & 0,17 & 0,32 & 0,54 & 1,00 & 0,43 & 0,68 & 0,01 & 0,38 & 0,09 \\
\hline (3) & & & & & & & 1 & 1 & 1 & $-0,33^{*}$ & 0,01 & 0,12 & $-0,05$ & $-0,30$ & 0,14 & 0,14 & $-0,19$ & $-0,50^{*}$ \\
\hline (a) & & & & & & & & & & 0,00 & 0,93 & 0,45 & 0,70 & 0,04 & 0,38 & 0,22 & 0,22 & 0,00 \\
\hline (4) & & & & & & & & & & 1 & 1 & 1 & 0,27 & 0,18 & $-0,06$ & $-0,01$ & $-0,06$ & 0,15 \\
\hline (a) & & & & & & & & & & & & & 0,02 & 0,24 & 0,69 & 0,94 & 0,70 & 0,34 \\
\hline (5) & & & & & & & & & & & & & 1 & 1 & 1 & 0,13 & $-0,36$ & $-0,47^{*}$ \\
\hline (a) & & & & & & & & & & & & & & & & 0,27 & 0,81 & 0,00 \\
\hline (6) & & & & & & & & & & & & & & & & 1 & 1 & 1 \\
\hline
\end{tabular}

Legenda:

10. Ano 2010; 11. Ano 2011; 12. Ano 2012

*. A correlação é significativa no nível 0,01 (2 extremidades).

(a) Sig. (2 extremidades)

Fonte: Dados da Pesquisa.

Observa-se na Tabela 3 que não existem correlações acima de 65,7\% com a variável dependente $\mathrm{AD}$, tampouco existem muitas correlações significativas entre as variáveis independentes durante os três anos analisados. Assim, com base na análise dessas correlações, é possível identificar que esses resultados indicam não haver multicolinearidade.

Os AD possuem correlação significativa e negativa com o Endiv em 2011 (54\%), em 2012 (65,7\%) e com a AlavFin em 2012 (30,5\%). Apresenta ainda correlação significativa e positiva com a AlavFin em $2010(35,9 \%)$ e em 2011 (42,2\%). Lin e Rong (2012) sustentam uma relação positiva entre os AD e o endividamento e alavancagem financeira, com base no fato de que empresas mais endividadas ou mais alavancadas teriam maiores incentivos para elevar seus resultados por meio dos AD. No entanto, neste estudo os achados confirmam tal resultado apenas para a alavancagem nos anos de 2010 e 2011.

O LnTam apresentou correlação significativa e positiva em 2010 com os ORA de 30,7\%. Constata-se, por meio da variável de controle tamanho, que as maiores companhias também registraram maiores valores de ORA em 2010. Conforme Lin e Rong (2012), a divulgação do ORA na Demonstração do Resultado irá melhorar a transparência das demonstrações contábeis, reduzir a assimetria da informação e, consequentemente, reduzir o gerenciamento de resultados. Essa correlação positiva também pode ser explicada pelo fato de que as transações registradas no ORA são típicas de empresas maiores, como ajustes em instrumentos financeiros, variação cambial de investimentos em controladas no exterior, entre outros.

O Endiv possui correlação significativa e negativa com a AlavFin em 2010 (33,4\%), com o FCO em 2011 (30,4\%) e com os ORA em 2012 (50,2\%). A variável FCO apresentou correlação significativa e positiva com a AlavFin em 2010 (27,4\%) e negativa com o ORA em 2012 (47,3\%). Com base em Lin e Rong (2012), esperava-se encontrar correlações significativas entre todas as variáveis independentes com o AD, entretanto, apenas o Endiv e a AlavFin apresentaram tal comportamento. Todas as variáveis também foram analisadas por meio da regressão de dados em painel e os resultados encontram-se na seção seguinte.

\subsection{Análise de regressão de dados em painel}

Para complementar a análise, aplicou-se a regressão de dados em painel sobre as variáveis do estudo a partir do software Stata $^{\circledR}$. Realizou-se no primeiro momento a análise dos pressupostos do modelo (efeitos fixos/ efeitos aleatórios), para verificar qual o modelo que mais se adaptava aos dados. Conforme Fávero et al. (2009), é possível obter, por meio da análise de dados em painel, três abordagens mais comuns: efeitos fixos, efeitos aleatórios e pooled independent cross-sections ou POLS (Pooled Ordinary Least Squares).

Aplicou-se também o teste de Chow (1960) que representa o teste F utilizado para determinar se os parâmetros de duas funções de regressão múltipla diferem entre si (FÁVERO et al., 2009). O teste F se mostrou 
significante ao nível de 5\%, o que fez com que o modelo POLS fosse rejeitado. Posteriormente, realizou-se o teste de LM de Breusch-Pagan a fim de verificar se o modelo aleatório poderia ser adequado para os dados analisados. Os resultados foram significantes ao nível de 5\%, o que indica a adequação do modelo de efeitos aleatórios. Para confirmar, aplicou-se o teste de Hausman, que indicou a não adequação ao modelo de efeitos fixos. Dessa forma, o modelo de efeitos aleatórios foi considerado o mais adequado para a análise dos dados. A Tabela 4 apresenta a análise de efeitos aleatórios.

Tabela 4. Análise de regressão de dados em painel

\begin{tabular}{lcll}
\hline Variáveis & Sinal Esperado & Coeficiente & VIF \\
\hline Constante & - & $0,9712823^{* * *}$ & \\
LnTam & + & $-0,0095727$ & 1,05 \\
Endiv & + & $-0,3734745^{* * *}$ & 1,07 \\
AlavFin & - & $0,0133574 * * *$ & 1,05 \\
FCO & - & $-0,5509945$ & 1,04 \\
ORA & & $\mathrm{DW}=1,6900$ & 1,08 \\
Durbin-Watson & & $\mathrm{SW}=0,9386$ & \\
Shapiro-Wilk & $\mathrm{F}=1,7142$ & \\
Levene & 0,1868 & \\
$\mathrm{R}^{2}$ & & 0,0007 & \\
Sig. & $\mathrm{F}=2,16$ & \\
F de Chow & $\mathrm{Sig}, \mathrm{F}=0,0004$ & \\
& $X^{2}=9,52$ & \\
LM de Breusch-Pagan & $\mathrm{Sig}, X^{2}=0,010$ & \\
Teste Hausman & $X^{2}=12,43$ & \\
\hline
\end{tabular}

*significativo no nível 0,1 (90\%); **significativo no nível 0,05 (95\%); ***significativo no nível a 0,01 (99\%)

Fonte: Dados da pesquisa.

A Tabela 4 descreve o modelo de regressão do período analisado (2010 a 2012). Constata-se que as variáveis independentes são eficazes para explicar as variações nos $\mathrm{AD}$, com significância ao nível de 0,01 . Verificou-se ainda que as variáveis independentes são responsáveis por 18,68\% da explicação dos AD.

O modelo de regressão não apresenta problemas de autocorrelação de primeira ordem entre os resíduos, uma vez que o teste de Durbin-Watson (DW) apresentou-se próximo de 2, de acordo com o indicado por Maroco (2003). Verificou-se por meio do teste de Shapiro-Wilk (SW) que a distribuição dos resíduos é normal (SW $=0,9386)$. A Homoscedasticidade foi testada aplicando-se o teste de Levene (F), que apontou uma variância uniforme dos erros $(\mathrm{F}=1,7142)$. Quanto à multicolinearidade, observou-se que o modelo apresenta Variance Inflation Factor (VIF) adequados para todas as variáveis, o que indica não haver problemas de multicolinearidade.

Destaca-se ainda que o presente estudo efetuou testes de robustez por meio dos modelos de Jones (1991) e Jones Modificado (1995). Tais modelos não apresentaram significância, o que corrobora com Martinez (2008) e Thomas e Zhang (2001) de que o modelo KS é o que proporciona os melhores resultados considerando a realidade brasileira.

Nesse sentido, a presente pesquisa encontrou como resultados uma relação negativa entre os ORA e os AD, conforme apontado na literatura (LIN; RONG, 2012), embora tal relação não tenha sido significativa. No entanto as variáveis com significância estatística sobre os AD foram Endiv e AlavFin. O Endiv apresentou efeito negativo, contrário ao esperado. Já a Alavancagem apresentou efeito positivo sobre os $\mathrm{AD}$, de acordo com o encontrado por Lin e Rong (2012). Observa-se na Tabela 2 (estatística descritiva) que a AlavFin aumentou consideravelmente em comparação ao Endiv no período analisado, ou seja, houve um aumento considerável de alavancagem financeira por meio de capital de terceiros, o que pode explicar os resultados encontrados nessa pesquisa.

O sinal dos coeficientes das variáveis LnTam (negativo), AlavFin (positivo) e ORA (negativo) seguiram o esperado, conforme o estudo de Lin e Rong (2012). Já as variáveis que não tiveram os sinais esperados foram: 
Endiv (com sinal negativo) e o FCO (com sinal positivo).

As pesquisas de Martinez (2001) e de Coelho e Lopes (2007), não encontraram significância para o efeito do endividamento no gerenciamento de resultados, ao contrário do observado neste estudo. Os resultados quanto ao efeito da variável AlavFin no gerenciamento de resultados, encontrados por Defond e Park (1997), foram negativos, já neste estudo observou-se uma associação positiva.

Esses resultados podem ser explicados, conforme apontam Lin e Rong (2012) pelo fato de que quanto maior o fluxo de caixa das atividades operacionais, menor é o accrual total, portanto, menor é o accrual discricionário. Por isso, esperava-se um coeficiente negativo para o fluxo de caixa das atividades operacionais, o que não se confirmou, entretanto, os coeficientes dessa variável não apresentaram significância.

Para Lin e Rong (2012), o tamanho da companhia é amplamente adotado para substituir o custo político, portanto, quanto maior a empresa, menor o grau de gerenciamento de resultados. Entretanto, neste estudo não foi encontrada significância estatística para esta variável.

\section{CONSIDERAÇÕES FINAIS}

O presente trabalho teve como objetivo verificar o impacto de Outros Resultados Abrangentes (ORA) no gerenciamento de resultados em companhias brasileiras listadas na BM\&FBovespa. Para tal, foi desenvolvida pesquisa descritiva, documental e com análise quantitativa, com as companhias brasileiras listadas na BM\&FBovespa no período de 2010 a 2013. No entanto, para a composição da amostra mantiveram-se as companhias com dados disponíveis no período de 2010 a 2012, conforme metodologia apontada na pesquisa.

Os resultados apontam que o tamanho da empresa, amplamente adotado para substituir o custo político (LIN; RONG, 2012), não apresentou significância com Accruls Discricionários (AD). Parte-se do pressuposto que, a fim de evitar custos políticos, os gestores muitas vezes tentam reduzir os lucros divulgados para não apresentar uma imagem de empresa excessivamente lucrativa perante o público. No entanto, esta situação não foi confirmada neste estudo.

O Fluxo de Caixa (FCO) também não apresentou significância. Considerando-se um determinado lucro líquido ou lucro operacional, um maior fluxo de caixa das atividades operacionais poderia sugerir menores provisões manobráveis (LIN; RONG, 2012), entretanto, neste estudo, não se confirmou esta situação.

Observou-se também que as variáveis com influência significativa nos Accruls Discricionários (AD) foram o Endividamento (Endiv), com sinal negativo, contrário ao esperado por Defond e Park (1997) e Lin e Rong (2012); e a Alavancagem Financeira (AlavFin), com sinal positivo, conforme encontrado por Lin e Rong (2012). Essas variáveis foram utilizadas como variáveis de controle por Lin e Rong (2012), por serem indicadores de desempenho e poderem influenciar na decisão de aumentar os Accruls Discricionários (AD) e gerenciar resultados, ou reduzir os accruals negativos, implicando na redução do lucro líquido.

Com base na regressão de dados em painel realizada, não se confirmou a relação entre o valor de Outros Resultados Abrangentes (ORA) e os níveis de gerenciamento de resultados para melhorar a qualidade das informações divulgadas. Assim, não se pode afirmar que essa divulgação abrangente aumentou a transparência das informações contábeis para a redução da assimetria informacional entre os stakeholders sobre o desempenho, ou para melhor compreensão sobre o resultado da empresa.

A divulgação de outros resultados abrangentes proporciona mais transparência por se tratar de mais detalhes na divulgação da demonstração de resultados, bem como fornece aos diversos stakeholders uma visão sobre o resultado da companhia mais a longo prazo. Quanto aos resultados deste estudo, mesmo não apresentando significância para a variável Outros Resultados Abrangentes (ORA), o que não permite fazer inferências sobre tais achados, constatou-se que o sinal do referido coeficiente para essa variável foi negativo. Isso significa que quanto maior os valores de resultados abrangentes, menor seriam os valores de AD.

Portanto, há um indicativo de que as empresas não utilizaram Outros Resultados Abrangentes (ORA) no período analisado para gerenciar seus resultados, o que implica dizer que a implementação da Demonstração de Resultado Abrangente (DRA) na contabilidade brasileira não propiciou aumento da assimetria informacional via Gerenciamento de Resultados. Assim, a visão de longo prazo em relação aos resultados da companhia fornecida pela DRA pode auxiliar os diversos stakeholders em suas tomadas de decisões. 
Por outro lado, como o sinal da variável ORA foi negativo, infere-se que companhias com valores elevados de Outros Resultados Abrangentes poderiam reduzir seus Accruls Discricionários (AD) no período atual para aumentá-los no momento seguinte, quando os valores de ORA forem reconhecidos no resultado.

No entanto, como não foi encontrada significância nos resultados para esta variável, não se pode afirmar que este comportamento esteja ocorrendo. Dessa forma, os resultados de Lin e Rong (2012) referentes à influência significativa de Outros Resultados Abrangentes (ORA) no gerenciamento de resultados não pode ser confirmada com este estudo no Brasil, pois não se obteve significância para essa variável testada.

Apresentam-se como limitação do estudo o modelos KS para detectar o gerenciamento de resultados por meio de accruals discricionários, uma vez que estes podem apresentar vieses pela dificuldade de estimação da discricionariedade dos accruals. Outra limitação é quanto a seleção da amostra, pois a conta Outros Resultados Abrangentes (ORA) foi apresentada apenas a partir de 2010, e nem todas as empresas divulgaram tal informação, podendo assim ocasionar viés de seleção de amostra. Tais limitações não invalidam os resultados alcançados por esse estudo.

Por fim, destaca-se que o estudo contribuiu com pesquisas já realizadas e com a discussão do conceito de resultado abrangente no Brasil, que é novo e ainda pouco discutido. Sugere-se que novas pesquisas possam ser realizadas com base neste tema, incluindo um período maior de análise, além de outras medidas de qualidade da informação contábil, como value relevance, por exemplo.

\section{REFERÊNCIAS}

BARTH, Mary E.; LANDSMAN, Wayne R.; LANG, Mark H. International Accounting standards and accounting quality. Journal of Accounting Research. v. 46, n. 3, p. 467-498, jun. 2008.

BIDDLE, Gary C.; CHOI, Jong-Hag. Is Comprehensive income useful? Journal of Contemporary Accounting \& Economics. v. 2, n. I, pp. 1-32, Jun. 2006.

COELHO, Antonio Carlos Dias; LOPES, Alexsandro Broedel. Avaliação da prática de gerenciamento de resultados na apuração de lucro por companhias abertas brasileiras conforme seu grau de alavancagem financeira. Revista de Administração Contemporânea, v. 11, n. SPE2, p. 121-144, 2007.

COELHO, Antonio Carlos; CARVALHO, L. N. Análise conceitual de lucro abrangente e lucro operacional corrente: Evidências no setor financeiro brasileiro. Brazilian Business Review. v. 4. N. 2. mai/ago, 2007.

COMITÊ DE PRONUNCIAMETOS CONTÁBEIS - CPC. CPC 26 (R1) - Apresentação das demonstrações contábeis. Disponível em: < http://www.cpc.org.br/mostraOrientacao.php?id=44> Acesso em: 01 dez. de 2013.

COMITÊ DE PRONUNCIAMETOS CONTÁBEIS - CPC. ICPC 10 - Interpretação sobre a Aplicação Inicial ao Ativo Imobilizado e à Propriedade para Investimento. Disponível em: < http://www.cpc.org.br/mostraOrientacao.php?id=70> Acesso em: 01 dez. de 2013.

DECHOW, Patricia M.; SLOAN, Richard G.; SWEENEY, Amy P. Detecting earnings management. The Accounting Review. v. 70, n. 2, p. 193-225, 1995.

DEFOND, Mark L.; PARK, Chul W. Smoothing income in anticipation of future earnings. Journal of accounting and economics, v. 23, n. 2, p. 115-139, 1997.

DEFOND, Mark; PARK, Chul W. Smoothing income in anticipation of future earnings. Journal of Accounting and Economics, n. 23, p. 115-39, 1997.

DHALIWAL, D; SUBRAMANYAM, K. R.; TREVEZANT, R. Is comprehensive income superior to net income as a measure of firm performance? Journal of Accounting and Economics, v. 26. Los Angeles: October, 1999.

FÁVERO, Luiz Paulo Lopes; BELFIORE, Patrícia Prado; CHAN, Betty Lilian; SILVA, Fabiana Lopes da. Análise de dados: modelagem multivariada para tomada de decisões. Rio de Janeiro: Elsevier, 2009.

GALLON, Alessandra Vasconcelos; SILVA, Tarcísio Pedro da; TOLEDO, Jorge Ribeiro de; HEIN, Nelson. Análise do ROA sobre as diferentes formas de apresentação do lucro nas empresas do nível 1 de governança corporativa da Bovespa. BASE - Revista da Administração e Contabilidade da Unisinos, v. 6, n. 1, pp. 
49-58, jan./abr., 2009.

HEALY, Paul M. The effect of bonus schemes of accounting decisions (Symposium on Management Compensation and the Managerial laboratory Market). Journal of Accounting \& Economics. Rochester, v. 7, p. 85$107,1985$.

HEALY, Paul M; WAHLEN, James M. A Review of the Earnings Management Literature and its Implications for Standard Setting. Accounting Horizons, vol.13, n. 4, p. 363-383, December, 1999.

HENDRIKSEN, Eldon S.; VAN BREDA, Michael F.; Teoria da contabilidade. Tradução de Antonio Zoratto Sanvicente. São Paulo: Atlas, 1999.

IUDÍCIBUS, Sérgio de; LOPES, A. B. (Coords). Teoria avançada da contabilidade. São Paulo: Atlas, 2004.

IUDÍCIBUS, Sérgio de; MARTINS, Eliseu; GELBCKE, Ernesto Rubens; SANTOS, Ariovaldo dos. Manual de contabilidade societária: aplicável a todas as sociedades: de acordo com as normas internacionais e do CPC. São Paulo: Atlas, 2010.

JONES, Jeniffer J. Earnings management during import relief investigations. Journal of Accounting Research. Oxoford. v. 29, n. 2, p. 193-228, aut. 1991.

KANG, S. H.; SIVARAMAKRISHNAN, K. Issues in testing earnings management: an instrumental variable approach. Journal of Accounting Research, Rochester, 33 (2) p. 353-367, 1995

KANG, Sok-Hyon; SIVARAMAKRISHNAN, K. Issues in testing earnings management and an instrumental variable approach. Journal of Accounting Research. v. 33, n. 2, Autumn, 1995.

KLANN, Roberto Carlos. Gerenciamento de resultados: análise comparativa de empresas brasileiras e inglesas antes e após a adoção das IFRS. 2011. 371 f. Tese (Doutorado em Ciências Contábeis e Administração) - Programa de Pós-Graduação em Ciências Contábeis da Universidade Regional de Blumenau, Blumenau, 2011.

KOCHIYAMA, Takuma; ITOU, Kunio. Does Comprehensive income influence dividends? $36^{\circ}$ Congress Annual $^{\circ}$ European Accounting Association. Paris, France: 2013.

LIN, Wang; RONG Men. Impacts of other comprehensive income disclosure on earnings Management. Nankai Business Review International. v. 3, n. 1, pp. 93-101, 2012.

MARTINEZ, Antonio Lopo. Gerenciamento dos resultados contábeis: estudo empírico das companhias abertas brasileiras. 2001. Tese (Doutorado em Ciências Contábeis) - Faculdade de Economia, Administração e Contabilidade - FEA. Universidade de São Paulo, SP, Brasil, 2001.

MARTINEZ, Antonio Lopo. Minimizando a variabilidade dos resultados contábeis: estudo empírico do income smoothing no Brasil. Revista Universo Contábil, ISSN 1809-3337, Blumenau, v. 2, n. 1, p. 09-25, jan./abr. 2006.

MARTINEZ, A.L. Detectando Earning Management no Brasil: Estimando os Accruals Discricionários. Revista de Contabilidade e Finanças - USP, São Paulo, v. 19, n. 46, p. 7 - 17, janeiro/abril, 2008.

MAZZIONI, Sady; ORO, Ieda Margaret; SCARPIN, Jorge Eduardo. Lucro versus resultado abrangente como medida preditiva do desempenho das empresas do setor elétrico brasileiro. Registro Contábil, v. 4, n. 3, p. 89-104, 2013.

PAULO, Edilson; LEME, Jéssica Rodrigues. Gerenciamento de resultados contábeis e o anúncio dos resultados contábeis pelas companhias abertas brasileiras. Revista Universo Contábil, v. 5, n. 4, p. 27-43, 2009.

PINHEIRO, Laura Taboada; DE MACEDO, Rodney Pereira; VILAMAIOR, Adriana Giarola. Lucro líquido versus lucro abrangente: uma análise empírica da volatilidade. Revista Universo Contábil, v. 8, n. 4, pp. 06-18, 2012.

SOUTES, D. O.; SCHVIRCK, E. Formas de mensuração do lucro e os reflexos no cálculo do ROA. Brazilian Business Review. v. 3, n. 1, pp. 74-87, jan/jun. 2006.

THOMAS, Jacob; ZHANG, Xiao-jun. Identifying unexpected accruals: a comparison of current approaches. Journal of Accounting and Public Policy, v. 19, n. 4, p. 347-376, 2001. 